\title{
High Efficiency Solar Cells via Tuned Superlattice Structures: Beyond 42.2\%
}

\author{
AC Varonides \\ Physics \& Electrical Engineering Dept, University of Scranton, Scranton, PA,
}

USA

\section{Introduction}

Modern PV devices are a direct outcome of solid state devices theory and applications of the last forty years. They are devices made of crystalline structures and basically, when illuminated with solar light, they convert solar photons into electric current. In the following a quick explanation of how this happens is presented. What is a solar cell? What is the basic function behind a cell's operation? Typically, in an illuminated p-n junction, photons are absorbed and electron-hole pairs are generated. These carriers diffuse in opposite directions (separated by the existing electrostatic field at the junction), and within their respective diffusion lengths. Electrons at the p-side diffuse through the junction potential and holes (similarly) get to the opposite directions. Under open-circuit conditions, the voltage across the cell is given by the following formula:

$$
V_{o c}=k T \ln \left(1+\frac{I_{L}}{I_{o}}\right)
$$

Where k is Boltzmann's constant, $\mathrm{T}$ (in Kelvin) is the cell temperature, $\mathrm{I}_{\mathrm{L}}$ is the lightgenerated current, and $I_{o}$ is the p-n junction's reverse saturation current (see below). Cell theory and $\mathrm{p}-\mathrm{n}$ junctions under a bias are briefly discussed in the next section.

\section{Background theory: The p-n junction}

Photonic device (solar cells included) operation is based on a p-n junction: two regions of the semiconducting material doped $\mathrm{p}$ and $\mathrm{n}$ type respectively and brought together in contact form a p-n junction. At thermal equilibrium, the p-n dope bulk semiconducting crystal, in order to keep its equilibrium, develops an internal field and develops its own built-in potential; the latter is total due to $\mathrm{p}$ - and n-type carrier migration across the junction.

Donor and acceptor atoms embedded in the lattice of the host material provide electrons and holes (as potential current carriers) that are free to wander in the crystal. In principle these carriers move randomly in the lattice, however, guiding these carriers accordingly could lead to non-zero currents coming off such semiconductors, and therefore to current producing devices. A semiconductor sample doped with donors and acceptors becomes a p$\mathrm{n}$ junction and therefore a device with two regions tending to overlap at their boundary. 
If the interface is at (say) $x=0$ position, free electrons and free holes diffuse through the interface and inevitably form space charge regions as shown figure 1 below:
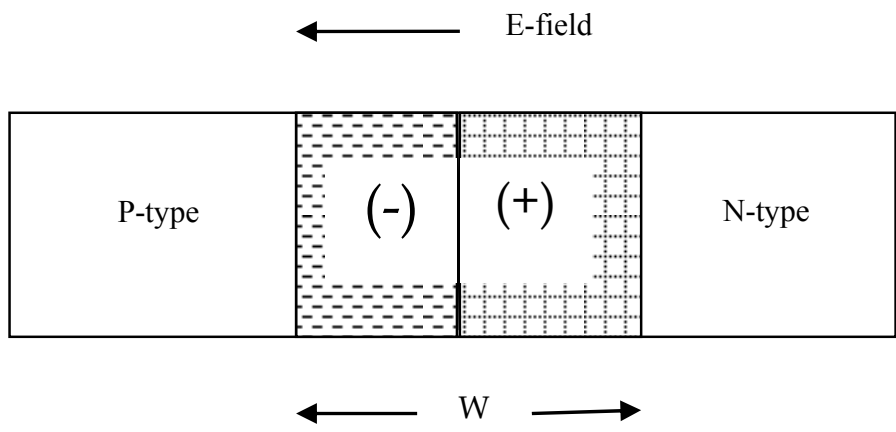

Fig. 1. pn-junction (e.g. of a Si sample) with the depletion $W$ region shown: both sides of the interface are shown, with their space charge distributions respectively.

A static electric field develops at the interface (figure above) emanating from the $(+)$ region and prohibiting respective carriers to further access the PN regions. From basic pnjunction theory, we can solve for the electric field and the potential developed by means of Poisson's equation. If the limits of the depletion region are $-x_{p}$ and $x_{n}\left(W=x_{p}+x_{n}\right)$ respectively, we can derive expressions for both field and potential developed at the junction:

$$
\left.\begin{array}{cc}
E(x)=\frac{q N_{d}}{\varepsilon}\left(x-x_{n}\right) & 0<x<x_{n} \\
E(x)=-\frac{q N_{a}}{\varepsilon}\left(x+x_{p}\right) & -x<x<0
\end{array}\right\}
$$

Where maximum field value is $E_{\max }=E($ at $\mathrm{x}=0)=-\left(q N_{d} / \varepsilon\right) x_{n} ; \mathrm{q}$ is the electronic charge, $\mathrm{N}_{\mathrm{d} \text {,a }}$ stands for donor and acceptor atom concentrations (per volume) respectively, $\varepsilon$ is the total sample's dielectric constant (or the product of the relative times the free space dielectric constants, e.g. $\varepsilon_{\mathrm{r}}=11.7$ for $\mathrm{Si}$ ). Based on expressions $(2,3)$ and on the fact that potential generated at the junction is the negative integral of the electric field across the depletion region, we can in principle derive the potential $\mathrm{V}(\mathrm{x})$ across the junction: it can be shown that $\mathrm{V}(\mathrm{x})$ is as follows:

$$
\begin{gathered}
V(x)=\frac{q N_{a}}{2 \varepsilon}\left(x+x_{p}\right)^{2} ; \text { In the p-region, and } \\
V(x)=-\frac{q N_{d}}{2 \varepsilon}\left[\left(x-x_{n}\right)^{2}-x_{n}^{2}\right]+\frac{q N_{a}}{2 \varepsilon} x_{p}^{2} ; \text { In the n-region and }
\end{gathered}
$$




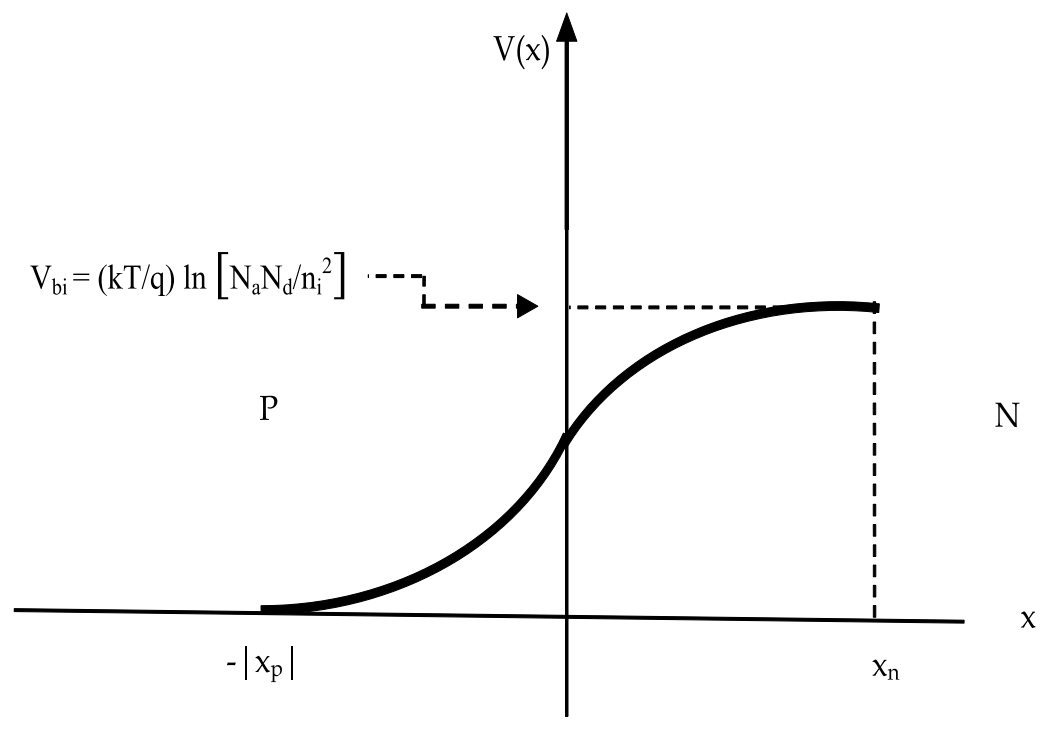

Fig. 2. Potential $V$ as a function of $x$ across the depletion region. Note (a) the two branches of $\mathrm{V}$ across both sides of the junction's boundary $(x=0)$ in accordance with (4) and (5) (b) the built-in voltage $V_{b i}$ at the right edge of the junction $[1,2,3]$. Note also that built-in voltage is normally computed as shown in the inset.

It is a straightforward matter to produce explicit results about widths in the junction area $\left(\mathrm{w}, \mathrm{x}_{\mathrm{n}},-\mathrm{x}_{\mathrm{p}}\right)$ in terms of device doping levels and built-in voltage values. The built-in voltage is determined from (4) at $x=x_{n}$ :

$$
V_{b i}=V\left(x=x_{n}\right)=\frac{q}{2 \varepsilon}\left(N_{a} x_{n}^{2}+N_{d} x_{p}^{2}\right)
$$

\section{Diode currents}

The fundamental current equation for $\mathrm{p}-\mathrm{n}$ junctions is derived based on considering that the built-in voltage is reduced down to $V_{b i}-V_{a}$, by the forward bias voltage $V_{a}$, helping majority carriers to escape and diffuse in the neighboring regions while, once electrons and holes reach the edges of the depletion region to the $\mathrm{p}$ and $\mathrm{n}$ regions, they diffuse accordingly according to a decaying exponential law of the type $\exp \left(\mathrm{x} / \mathrm{L}_{\mathrm{n}, \mathrm{p}}\right)$; the latter includes distance $x$ and the diffusion length for electrons and/or holes respectively. Excess minority carriers diffuse in both regions according to the following expressions:

$$
\begin{gathered}
\delta p(x)=p_{n o}\left(e^{x / L}-1\right) e^{\left(x+x n_{n}\right) / L p} \\
\delta n(x)=n_{p o}\left(e^{x / L} p-1\right)
\end{gathered}
$$


Where $\mathrm{p}_{n o}$ is holes in the n-region, $\mathrm{L}_{\mathrm{p}}$ is the diffusion length of holes in the n-region, and where $\delta p$ represents excess holes in the n-region. Diffusion currents can be calculated by means of the diffusion equation along with suitable boundary conditions:

$$
\begin{aligned}
& J_{p}=-q D_{p} \frac{d \delta p(x)}{d x} \mid \quad\left(\mathrm{x}=\mathrm{x}_{\mathrm{n}}\right) \\
& J n=q D_{n} \frac{d \delta p(x)}{d x} \mid \quad\left(\mathrm{x}=-\mathrm{x}_{\mathrm{p}}\right)
\end{aligned}
$$

Based on the above expressions, current density of the p-n junction due to a forward bias $V_{a}$ is found to be as follows (see also (1)):

$$
J=J_{o}\left(e^{V a / V t)}-1\right)
$$

(Where $\mathrm{V}_{\mathrm{t}}$ is the thermal voltage $(\mathrm{kT} / \mathrm{q})$ )

\section{4. p-n junctions as solar cells}

Fundamentally, solar cell modeling correlates incident solar photon flux $\Phi_{\text {ph }}$ (\# of photons $\mathrm{cm}^{-2} \mathrm{~s}^{1}$ ) with generation and recombination carrier rates in the interior of the device. Photogenerated concentrations of diffusing carriers are typically modeled through the diffusion equation (under appropriate boundary conditions):

$$
\frac{d^{2} \delta p_{n}}{d x^{2}}-\frac{\delta p_{n}}{L_{p}}+\alpha(1-R) \Phi_{p h} e^{-\alpha(x+d)}=0
$$

Photon-collection efficiency is usually defined as the ratio of total current over solar photoflux $\left(\mathrm{cm}^{-2} \mathrm{~s}^{-1}\right)$ :

$$
\eta_{c o l}=\frac{J_{p}+J_{n}-J_{r e c}}{q \Phi_{p h}}
$$

The numerator in (13) is total photo-induced current in the $\mathrm{p}$ and n-regions minus recombination current. Boundary conditions include continuity of carrier concentrations at the junction $x(j)$, and the dependence of the first derivative of carrier concentration on recombination velocity $s_{p}$, at the edge of the window layer as shown in the figure below:

$$
\left(\frac{d \delta p}{d x}\right)_{x=-d}=\frac{s_{p}}{D_{p}}\left(p(-d)-p_{n o}\right) \cong \frac{s_{p}}{D_{p}}(p(-d))
$$

Figure-3 shows a generally accepted modeling geometry of a p-n junction solar cell. These two regions are separated by the depletion region (of thickness $\mathrm{w}$ ): majority electrons from the n-region migrate to the p-region, and majority holes reciprocate from the latter region. 


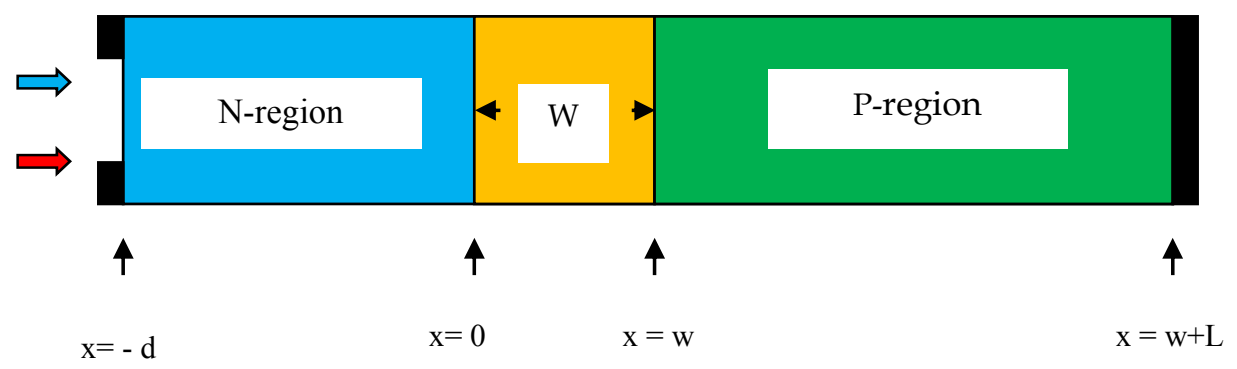

Fig. 3. Typical modeling geometry of a solar cell: $\mathrm{w}$ is the depletion width, $\mathrm{J}$ is the exact interface, $\mathrm{L}$ is the width of the p-region and $\mathrm{d}$ is the n-region (window layer). Note that the $\mathrm{n}$-region is the window for solar photons.

Minority holes generated in the window layer ( $\mathrm{x}$ from $-\mathrm{d}$ to 0 ) are:

$$
\delta p_{n}(x)=C \exp \left(-x / L_{p}\right)+\frac{\alpha \Phi_{p h}(1-R)\left(L_{p}^{2} / D_{p}\right)}{1-\left(\alpha L_{p}\right)^{2}} e^{-\alpha(x+d)}
$$

Note that at $\mathrm{x}=0: \delta p_{n}(0)=\frac{\alpha \Phi_{p h}(1-R)\left(L_{p}^{2} / D_{p}\right)}{1-\left(\alpha L_{p}\right)^{2}} e^{-\alpha(x+d)}$

Maximum hole- current density generated in the n-region is:

$$
J_{p}(x=0)=-q D_{p} \times\left[\begin{array}{l}
\frac{1}{L_{p}}\left(\frac{\alpha F_{p h}(1-R)\left(L_{p}^{2} / D_{p}\right)}{\left(\alpha L_{p}\right)^{2}-1}\right) \times \\
{\left[\tanh \left(\frac{d}{L_{p}}\right)+\frac{\alpha L_{p}}{\cosh \left(d / L_{p}\right)}+\frac{s_{p} L_{p}}{D_{p}} \times \frac{p(-d)}{\cosh \left(d / L_{p}\right)}\right]+\alpha e^{-\alpha d}}
\end{array}\right]
$$

The surface recombination velocity $s_{n}$ at the edge of the p-region is

$$
-D_{n}\left(\frac{d n}{d x}\right)_{x=L+W}=s_{n}\left(n_{p}-n_{p o}\right)
$$

The diffusion equation reads as follows:

$$
\frac{d^{2} n_{p}}{d x^{2}}-\frac{n_{p}-n_{p o}}{L_{n}}+\alpha(1-R) \Phi_{p h} e^{-\alpha(x+d)}=0
$$

Solution of (18) is of similar kind with (12) along with boundary conditions (17):

$$
\delta n(x)=A \cosh \left(x / L_{n}\right)+B \sinh \left(x / L_{n}\right)+\frac{\alpha \Phi_{p h}(1-R)\left(L_{n}^{2} / D_{n}\right)}{1-\left(\alpha L_{n}\right)^{2}} e^{\alpha(x+d)}
$$


The total current out of the cell is the sum of all currents minus recombination components from each region, especially recombination at the $\mathrm{w}$ region. Excess carriers in solar cells (as in any photonic device) are minority electrons and holes in the $\mathrm{p}$ and $\mathrm{n}$ regions respectively. When a cell is illuminated, solar photons excite electron hole pairs in all regions: the p-, nand depletion regions. The latter may be become of great significance for the following reason: excited electrons and holes do split away from each other due to the existing electrostatic field. This means that these excess carriers will reach the edges of the depletion region in a very short time. Note also that typically, mean diffusion lengths of these carriers are much longer than $t$ he actual width of the depletion area (even in pin devices). This makes the depletion region especially attractive for illumination: electrons and holes will separate from each other quickly, and they will diffuse in the bulk parts of the cell very fast assisted by the electrostatic field. In addition, space availability in the mid-region provides a chance for excess layer $\mathrm{s}$ that can be tuned to desired solar photons for subsequent absorption, thus enhancing device performance. This is why multi-layers are used in the intrinsic region (long depletion region in $p-n$ junctions). If tuned quantum wells are grown somewhere in the middle, incident solar illumination will push electrons in the quantum wells and to tunneling or thermionic escape. The notion of additional band gaps integrated in the intrinsic region has been adopted successfully recently. For instance, successful cells with more than one band gaps have been designed and realized, where two or three cells are connected in series forming tandem cells with the advantage of voltage increase. This is possible due to the series connection of the tandem cells. Tandems provide excess voltage but they lack in current, in other words, due to the differences of the layers involved, current matching will be enforced due to the series connection. If these structures can ensure relatively high current outputs, then, along with increased voltage one should expect efficiency improvements. In the next we outline the behavior of a cell in tandem: top cell of AlAs/GaAs and bottom cell of a pin GaAs/Ge/Alloy for long wavelengths.

\section{Heterojunction cells}

Improved cell design has to include more than one band-gap for larger number of absorbed photons. P-i-n (from now on pin diode) diode designs offer wide intrinsic regions between the $\mathrm{p}$ - and $\mathrm{n}$ - regions of a $\mathrm{p}-\mathrm{n}$ junction, where photo-carriers have a great chance to be generated and quickly swept away to the ends of the two-lead diode. This is possible due to the electrostatic field that develops at the depletion region. Illumination of the structure at the intrinsic region or a pin increases the chances of more photo-excited carriers. On the other hand, for a pin diode exposed to solar light and with a thin p-layer, minority electrons from the p-region may cross very fast $\left(\tau_{n} \sim\right.$ fraction of $\mu$ s) the junction at the $p$-i interface and be swept away to the load by the electric field in the mid-region of the cell. More than one band gaps in the mid region may lead to quantum wells where quantum size effects may take over as long as thickness values are in the order of 5 to $8 \mathrm{~nm}$. Superlattice-like structures may be grown in the intrinsic region in order to accommodate both short and long solar wavelengths. It is commonly accepted that thin bulk window layers grown on top of a wide mid-region with quantum wells may offer a two-fold advantage (a) short wavelengths absorbed at the top and longer wavelengths absorbed in the mid region where a superlattice structure is essentially tuned at specific wavelengths. Thus, by growing a superlattice in the middle of a pin region (rather by changing the mid-region into a multiquantum well (mqw) sequence) one may reach the main objective: to capture more solar 
photons with energies higher than the band gap of the host material. The figure below depicts an intrinsic multi-quantum well area, where discrete energy levels cause a widening of the host material's gap (commonly GaAs with gap at $1.42 \mathrm{eV}$ ).

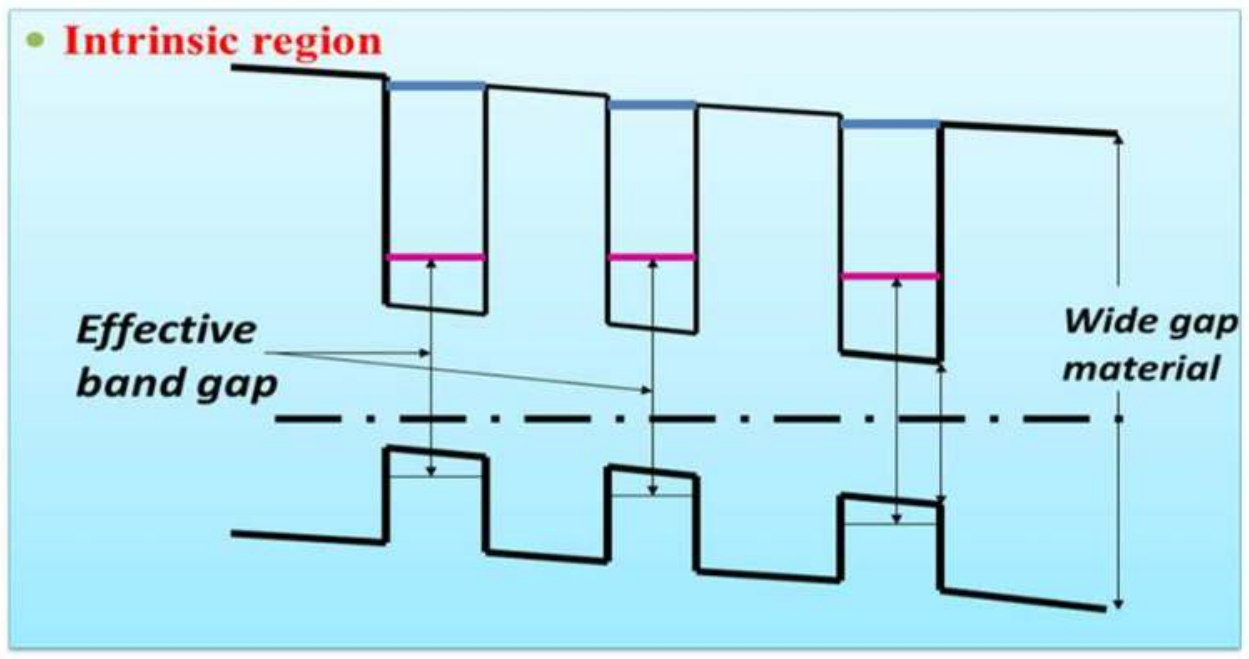

Fig. 4. Detail from a superlattice structure (typically GaAs/alloy and GaAs/Ge (as proposed in this study). The dashed line represents the Fermi level at thermal equilibrium. The optical gap can be tuned to desired energy values.

Figure 5 shows a superlattice covering the mid region of a pin cell:

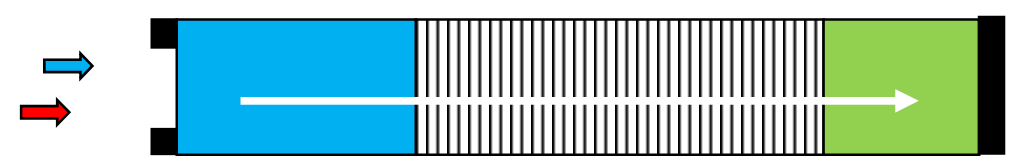

Fig. 5. A p-i-n GaAs/alloy superlattice developed in the mid-region of a pin cell: the middle section depicts: the top layer (blue) is the wide gap alloy (e.g. AlAs) and the bottom layer is GaAs (host material); GaAs is also grown in the superlattice as the low gap medium.

Such a cell design (shown above) is an expanded p-n junction with a wide superlattice midregion occupying the intrinsic or low doped region between $\mathrm{p}$ and $\mathrm{n}$. To reduce cost such a structure can be compromised by inserting a short period tuned superlattice as a small percentage of the device as a total.

\section{Top cell (AIAs/GaAs)}

Modeling of the top region may be performed in two ways, by considering the equivalent circuit of the device and/or by solving for excess carriers and subsequent electric currents and current densities in the solid state. In this brief outline we are considering the first 
approach by starting from the basic illuminated diode equation and adopting standard results regarding maximum power, short circuit current and open-circuit voltage values. Starting from the fundamental solar cell equation, we can derive maximum power conditions:

$$
I_{m}=\frac{\beta V_{m}}{1+\beta V_{m}} I_{L}
$$

Where $I_{m}, V_{m}$ are maximum current and voltage values, and where $\beta=q(k T)^{-1}$.

And

$$
V_{m}=V_{o c}-\frac{1}{\beta} \ln \left(1+\beta V_{m}\right)
$$

Efficiency (as power out over power in) is shown to be:

$$
\eta=\frac{P_{o}}{P_{i n}}=\frac{I_{L} V_{o c}}{P_{i n}}\left[1-\left(\frac{V_{m}}{V_{o c}}\right) \frac{\ln \left(\beta V_{m}\right)}{\beta V_{m}}\right]
$$

It is clear from (22) that the quantity in brackets is the fill factor (FF) of the device which is found based on maximum voltage values and open circuit voltage:

$$
F F=1-\frac{\ln \left(\frac{V_{m}}{k T}\right)}{\left(\frac{V_{o c}}{k T}\right)}=1-\frac{\ln \left(\beta V_{m}\right)}{\beta V_{o c}}
$$

Highly efficient solar cells have been found to have open-circuit voltages within a range from 1 to $1.08 \mathrm{~V}$. The table below indicates how open circuit voltage controls maximum voltage (voltage at maximum power point). Assuming short circuit current at $30 \mathrm{~mA} / \mathrm{cm}^{2}$, under one-sun $\left(100 \mathrm{~mW} / \mathrm{cm}^{2}\right)$, the efficiency is depicted below by Table 1 :

\begin{tabular}{|c|c|c|c|}
\hline $\mathrm{V}_{\mathrm{oc}}(\mathrm{V})$ & $\mathrm{V}_{\mathrm{m}}(\mathrm{V})$ & $\mathrm{FF}(\%)$ & $\eta(\%)$ \\
\hline 1.02 & 0.926 & 0.907 & 27.75 \\
\hline 1.03 & 0.938 & 0.906 & 27.99 \\
\hline 1.04 & 0.948 & 0.905 & 28.23 \\
\hline 1.05 & 0.958 & 0.904 & 28.50 \\
\hline
\end{tabular}

Table 1. Open circuit and maximum voltages, Fill Factor (FF) and collection efficiency (300 Kelvin). The cell is the top AlAs/GaAs that serves as a window to the solar flux.

It is of advantage to suggest an undoped GaAs-Ge multi-quantum well (MQW) in a standard pin-design, namely, p-intrinsic (MQW)-n geometry that includes lattice-matched GaAs and Ge layers in the intrinsic region of the PV device. This formation could offer the advantage of $1 \mathrm{eV}$ absorption (at the appropriate quantum well width), without compromises in device transport properties, such as mobility or conductivity. GaAs-based layers provide (a) high mobility and absorption values and (b) a chance for fine-tuning of the optical gap with specific solar photon wavelength. Recently, high efficiency cell designs have been proposed where two pn cells are grown in tandem (series connection), where the top 
cell is a bulk (e.g. GaAs/AlAs cell) and the bottom is a superlattice-based pin cell optimized at long wavelengths. Such devices offer efficiency increase by acting simultaneously: top unit near 20\% and bottom unit near 30\% (when they operate on their own) lead to structures (quantum cells) with overall efficiencies in excess of $35 \%$ (under one sun and with recombination effects and scattering included). As seen in Figure 5 below, the superlattice approach offers a tool for capturing solar photons at desired wavelengths with the appropriate quantum mechanical tuning. In other words, ground eigen-states in quantum wells match specific wavelengths (corresponding to photons with the same energy);

\section{Solar Radiation Spectrum}

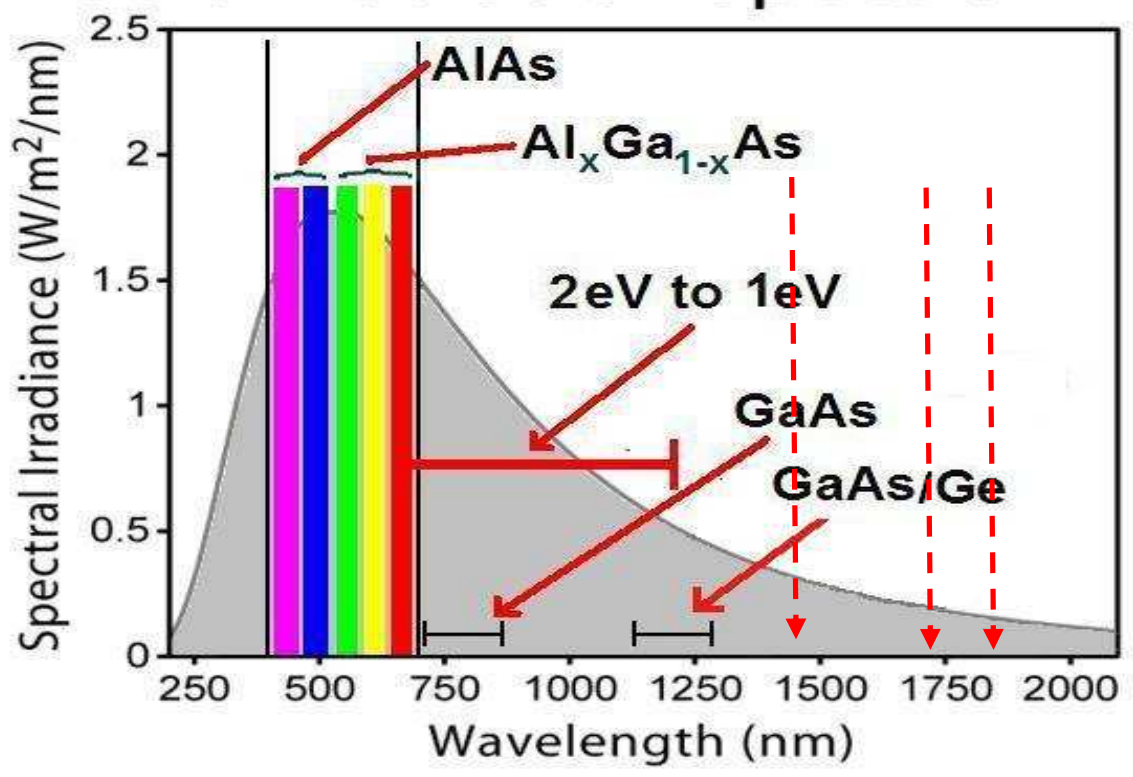

Fig. 6. Regions of the solar spectrum covered by the superlattice cell and the top cell (visible). The superlattice can be tuned at $\sim 1 \mathrm{eV}$. Dashed arrows indicate region of feasible absorption from the superlattice region

As seen from the figure above, almost full spectrum absorption can be achieved with materials that absorb at desired photon energies. Specifically, visible photons may be absorbed by means of a GaAs/AlAs bulk cell, and IR radiation absorption can be achieved via GaAs $(1.42 \mathrm{eV})$ and $\mathrm{Ge}(0.67 \mathrm{eV})$ respectively with superlattice or superlattice sections tuned at desired wavelengths. The n-region of the pin cell can be selected to be Ge in the bulk, ensuring absorption at the tail of the solar spectrum (for Ge: wavelength absorbed at $\lambda=1.24 / 0.67=1.85 \mu \mathrm{m}$, see last arrow in the figure above). How is the current formed in the superlattice layer? The answer hides in the quantum nature of this region: quantum wells quantized the energy of the captured electrons (and light and heavy holes in the valence band); photo-excited electrons escape thermionically from the wells and form excess current in the conduction band. On the other hand, incident IR photons are expected to be absorbed in the MQW area. Projected excess carrier population (electrons with recombination 
included) is of the order of $10^{12}$ to $10^{13} \mathrm{~cm}^{-2}$ per eigen-state. Thermionic current density values have been found to be near order of $30 \mathrm{~mA} / \mathrm{cm}^{2}$ and open-circuit voltage values above $1 \mathrm{~V}$, at one sun. Overall (for a composite cell see figure 3 ) collection efficiency values are initially projected well in excess of $35 \%$, which is a key for immediate improvement to even higher collection efficiency. Total current density is dominated by the lowest of the two sub-cell currents, and open-circuit voltage values are the sum of the two sub-cell $V_{\text {oc }}$ values. Total current from the bottom cell is the sum of thermionic and nearest neighbor hopping currents. Preliminary results reach estimates of efficiencies from each of the two (latticematched) sub-cells in excess of $21 \%$ per cell (predicted synergy of the two sub-cells in excess of $40 \%$ ). Loss mechanisms at interfaces and quantum wells and their role in overall efficiency determination will also be included. Advantages of the design are:

i. Solar spectrum matching in both visible and IR ranges through layer band gapmatching selection.

ii. Lattice-matching

iii. Increased carrier transport due to GaAs. It is conceivable that even the $40 \%$-plus target of conversion efficiency can be reached with such designs

Heterostructure and (most recently) multijunction solar devices exhibit better performance in transport properties, when compared to bulk solar cells: especially in quantum well devices, photo-excitation causes carrier accumulation in discrete energy levels, with subsequent escape to the conduction band (minus recombination losses) via standard mechanisms such as tunneling, thermal escape or nearest neighbor hopping conduction. Full spectrum absorption and triple junction solar cells have become key factors for high efficiency collection in PV structures of various geometries. Most recently, successful photovoltaic device (PV) designs have shown high efficiency values well above $30 \%$, and efficiency levels in excess of $40 \%$ have been reached by means of triple junction metamorphic solar cells and under high sun concentration (good candidate for concentrated PV or CPV). Multijunction solar cells offer a great advantage over their bulk counterparts: by incorporating lattice-matched alloys, one may succeed in designing a device with more than one energy gaps thus increasing the number of absorbed solar photons. During the last decade, various groups have modeled and developed multijunction solar cells in order to increase overall collection efficiencies. Emphasis has been given in two types of PV devices (a) lattice-matched solar cells and (b) metamorphic (lattice-mismatched) solar cells. In particular, III-V multijunction solar cells have shown the greatest progress in overall efficiency. The broader impact of this project is a new design proposal for high efficiency solar cells. The target is to exceed $45 \%$ collection efficiency for very efficient photovoltaic devices. It is more than clear that once such a cell is realized, the field of concentration photovoltaics (CPV) will benefit greatly: solar cells with (a) record high efficiency values (b) under several hundred suns (Fresnel optics at 500+ suns) and (c) small in size (low area hence less material) is already attracting interest for mass production in many places in the world. In recent years, it has been proposed by us a new design for a high efficiency and lattice-matched solar cell (HESC), where both visible and infrared portions of the solar spectrum are absorbed according to the structure's geometric material arrangement: simultaneous absorption of both short and long wavelengths. In this on-going research enterprise, the synergy between a highly efficient triple junction cell and a highly efficient superlattice or a multi-quantum well region, is presented as a new and innovative way for further efficiency increase. It is well established by now, that triple junction solar cells are exceeding the upper threshold of collection efficiency to ever higher levels, namely 
above 38\% with latest threshold at 41.1\% (Fraunhofer Institute, Germany). Currently, a cell that will operate above the $40 \%$ threshold is in target, with ultimate target the efficiency at or near $50 \%$. The cell design is based on a p-i-n bulk device model with three distinct areas, two of which are complete PV-heterostructures on their own; in other words, these two regions could stand alone as two independent solar cell structures with quite acceptable performance (of the order of $21 \%$ and more as it has been demonstrated by our group recently). The power output of the PV composite device is a function of the individual power outputs from each sub-cell in the PV unit. On the other hand, triple junction solar cells seem to lead the way to high efficiency photovoltaics especially in the area of concentrated photovoltaics (CPV), where small cell area and therefore less material (hence lower material costs) may lead to high PV performance. The latter are triple junctions of lattice-matched and non-lattice matched III-V heterostructures with two tunnel junctions between the layers.

\section{Suggestions for modeling}

Fully develop a theoretical model of PV composite PV devices by first principle calculations and computations based on realistic device parameters; propose a composite PV structure with two major cells: a triple junction and multi-layer tuned cell, with the prospect of high efficiency near $50 \%$. Modeling tools include several established math software packages. Seek for a composite photovoltaic device that combines properties of direct-gap crystalline semiconductors and absorption in the entire spectrum, mainly in the visible and in the infrared (NIR/IR) wavelength ranges, and which is configured as a two-part solar cell: a top triple junction and a multi-layer p-i-n bottom unit tailored to IR infrared wavelengths. The solar spectrum (a 6,000 $\mathrm{K}$, see in Figure 5) offers the option of finding suitable band gaps for highest absorption. Material selection shows a blue shift in the absorption via wide gap materials as shown (AlAs). Low gap materials offer wavelength matching in the IR range (note the dashed arrows indicating optical gaps corresponding to various wavelengths. It is of advantage to exploit quantum wells grown on n-type or low-doped substrates.

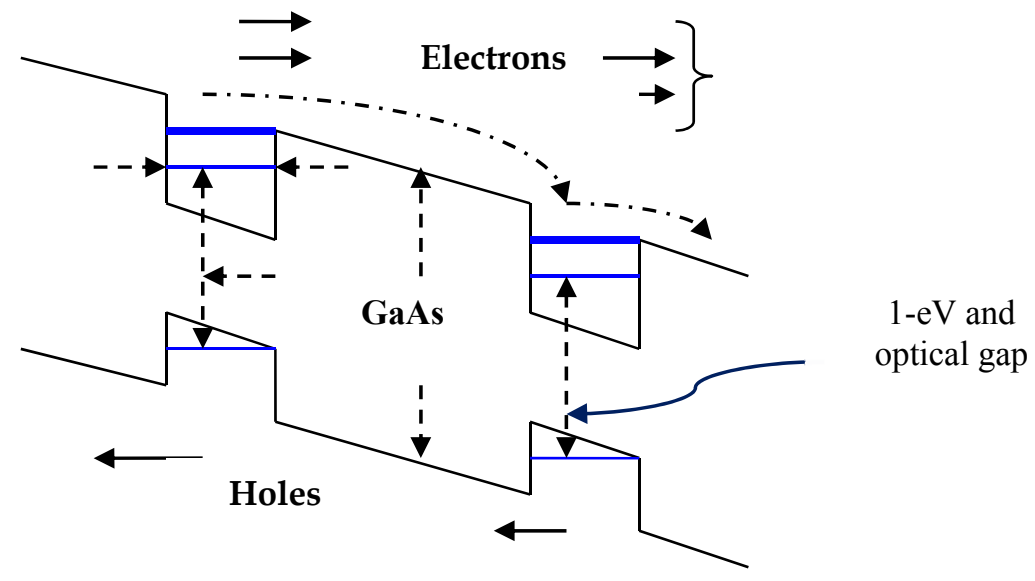

Fig. 7. Tuned quantum wells at $1 \mathrm{eV}$ solar photons: shown are energy levels and optical gap increase 
Superlattice structures in both cases mentioned above are at the designer's disposal, in the sense that appropriate quantum well geometries may lead to desired solar photons absorption. Enhancement of cell performance can be achieved by replacing the intrinsic region with tuned multi-quantum well (MQW) layers, designed for specific wavelengths. Thermionic emission, hopping conduction and tunneling are dominant mechanisms of photo-carrier transport in heterostructures (against losses due to recombination processes). Photo-excited layers thermally escape from quantum wells (minus recombination losses): incident solar photons typically generate $10^{12}$ to $10^{13}$ net photo-excited carriers per unit area $\left(\mathrm{cm}^{2}\right)$, after recombination effects have been taken into account. This population is expected to migrate to the conduction band assisted by the escape mechanisms named above and the built-in electrostatic field in the p-i-n region.

By selecting suitable geometry of the quantum wells that leads to one or two energy levels in the quantum wells, ground state of electron-hole pairs at $1 \mathrm{eV}$ may be formed, and a second state at the very edge of the GaAs layer conduction band (see Fig 2): this event has been shown to act in favor of nearest neighboring hopping electrons from site to site $(\mathrm{QW})$. Thus a three-fold advantage of the superlattice/MQW region is that (1) excess (in addition to carriers from the bulk part of the device) carriers are trapped and thermally escape to the conduction band and (2) nearest neighbor hopping conduction ( $\mathrm{NNH}$ ) becomes a second conduction mechanism and (3) band gaps of other materials may be represented via energy levels in quantum wells. The total current from the intrinsic region will be the sum of the thermionic and the $\mathrm{NNH}$ current components (minus recombination losses). Subsequent well width selection may lead to further refinement of solar photon absorption. Near infrared and infrared portions of the solar spectrum can be covered by suitable width selections, with equal amount of modeling effort (from the point of view of computations, it is a mere change of parameters for slightly different optical gaps). It is also interesting to note at this point that quantum well width could be modeled as a random variable, leading to a random distribution of optical gap values (as function of well width) and hence a smeared distribution of optical gap values and absorbed photon wavelengths, for the benefit of the photovoltaic device. Thus, IR photon absorption in the neighborhood of $1 \mathrm{eV}$ is feasible. In addition, the superiority of transport properties of the proposed quantum-PV device should be noted compared to its III-N-V "high" efficiency counterpart: our proposed superlattice cell is mainly a GaAs device perturbed by thin Ge layers, and therefore this region exhibits much higher electron mobility. In the absence of tunneling (thick potential barriers) total currents are in essence the sum of (a) bulk currents from the mainly bulk pin device (b) thermionic and (c) hopping current components, due to free electrons in the GaAs conduction band, assisted by the overall electrostatic field in the intrinsic region. Recent modeling and simulation have shown that the top cell retains visible absorption (AlAs/ (Al) GaAs/GaAs at 21\%) or to include a highly efficient triple junction cell (in this proposal, our own choice (InP/GaAs/GaAs at 30\% efficiency). The bottom multi-well cell operates at longer wavelengths $(1 \mathrm{eV}$ or $1,240 \mathrm{~nm})$, and therefore the whole of the unit absorbs in both regimes visible and IR respectively. Since germanium and gallium arsenide layers are lattice-matched, it is conceivable that a superlattice would fit in between the p- and nregions of the device. Advantages of such designs are summarized below:

1. less material to grow

2. small area (exposed to sun-light cell aperture) 
3. less complexity in the structure overall

4. reduced scattering of drifting and diffusing carriers

5. reduced carrier trapping and recombination (carriers in MQW region separate from their corresponding holes as being away from the quantum wells)

6. faster growth conditions attainable

7. lower fabrication costs

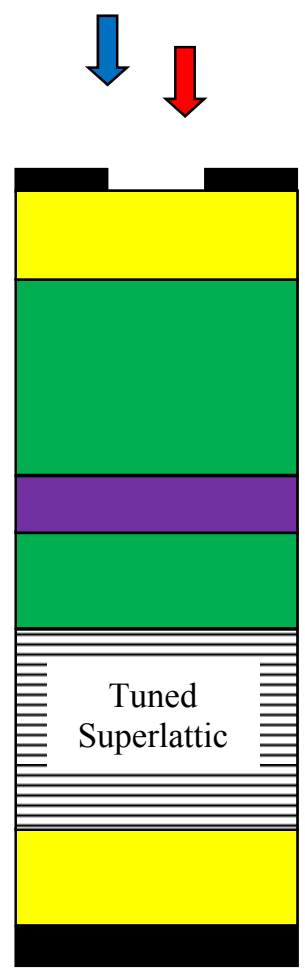

Fig. 8. Proposed cell structure: top cell p-n junction, tunnel junction (TJ) (purple) and p-i-n bottom cell with superlattice in the middle; P region (green), $\mathrm{N}$ region (yellow). Top cell is the window facing the sun (anti-reflected coating and surface texturing not shown).

As seen from the figure above, there are several options for further design and optimization (a) top region offers the possibility of another superlattice tuned at selected wavelengths (b) layers and alloys other than GaAs can be used (in the lattice-matched fashion) (c) tuned superlattice (bottom cell) can be split is more than one narrow units tuned at desired solar spectrum peaks (d) cell can be of small area (less material used) or of large area for higher exposure.

\section{Some thoughts on concentrated photovoltaics (CPV)}

Concentrated light on small solar cells can become of great advantage: a small size cell $\left(\sim 2 \mathrm{~mm}^{2}\right.$ area) may be placed at the focal point of a Fresnel optical system. Concentrated light causes higher carrier absorption from the bulk of the device and therefore higher 
current generation. Currently III-V multijunction cells have shown to have the highest collection efficiency. Efficiency $\eta(\%)$ increases logarithmically with solar power up to about 500 suns (one sun $=100 \mathrm{~mW} / \mathrm{cm}^{2}$ ). Currently, it seems that CPV cells show the highest efficiency (consistently above 38\%) with latest record efficiencies at $41.1 \%$ (Fraunhofer Institute). As it can be seen from equation (11) and the efficiency expression: $\eta(\%)=\left(V_{\mathrm{oc}} \mathrm{J}_{\mathrm{sc}} \mathrm{FF}\right) / \mathrm{P}_{\mathrm{in}}$, the efficiency of a solar cell increases logarithmically with $\mathrm{J}_{\mathrm{sc}}$. Such a behavior has been observed, in fact, $\eta(\%)$ increases with increasing current generation (maximum value in the neighborhood of 550 suns).

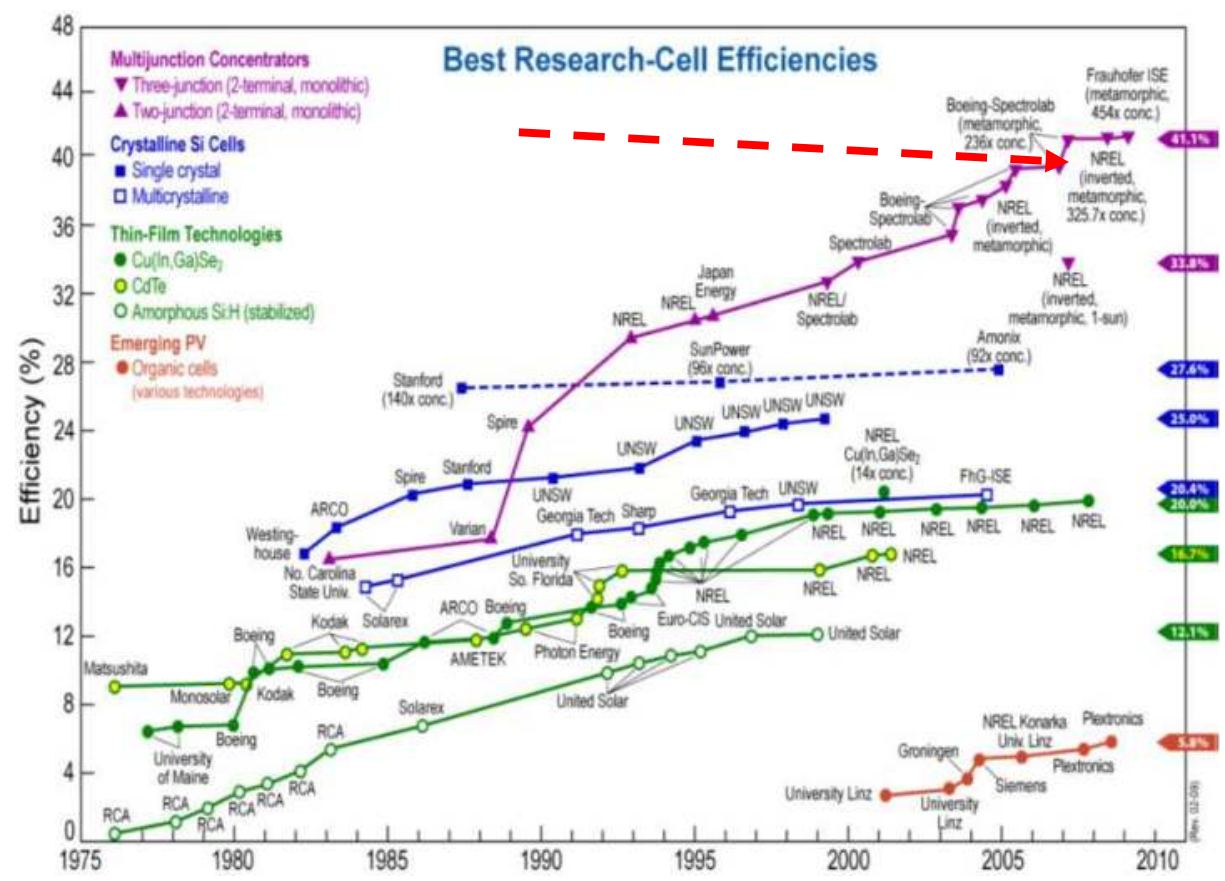

Fig. 9. Current status of cell performance and improvement since the mid seventies. Note that MJ cells have taken the lead in the high efficiency race. Latest (2010) results: $41.1 \%$ collection efficiency (Fraunhofer Institute at 454 suns) [@ 2009 Spectrolab, Inc. All rights reserved].

As seen from the figure above, multijunction cells, with more than one band gaps, take the lead in current and voltage production (recall that efficiency varies with open-circuit voltage and short-circuit current). CPV systems have given a boost of solar power production globally because they combine (a) highly efficient cells with small exposure area and (b) less costly optical system and components. As of 2009, CPV systems operate at $28-30 \%$ total efficiency (cell plus optics) and seem to be coming dynamically in the global PV market.

\section{Conclusions: The immediate future}

Photovoltaics is the child of progress in condensed matter physics, and has matured to the point that solar energy has been competing with fossil fuel energy sources. Small in size 
highly efficient solar cells are the answer for our future energy needs. The $40 \%$ threshold has already been reached and current research shows that $50 \%$ photovoltaics will soon be a reality. It seems that such high conversion will be succeeded by means of small size highly efficient solar cells. By small size we mean from the $\mathrm{mm}$ level down to nano-sized PV particles mounted to Fresnel-type optical systems with high solar concentration. Global energy production based on high efficiency PV will solve the energy needs of all nations and will slow down planet pollution. No nuclear waste and zero chance for accidents will guide common sense in immediate future. The concept of tuned superlattices was outlined and its advantages have been presented. Well-understood and lattice-matched materials, such as GaAs/Alloy and Ge, along with improved growth techniques pave the way to high efficiency photovoltaic devices. Integrated circuit techniques are also available for cells of minute size (e.g. $5 \mathrm{~mm}^{2}$ ), which is a dramatic reduction of material and hence of cost. Reduced size photovoltaic cells, under high solar concentration (currently from 450 to 500 suns), have opened the avenue for a competitive PV industry in the near future. Concentrated Photovoltaics (CPV-farms) will eventually dominate the world energy production. PV system price range has been steadily reducing from $\$ 0.40 / \mathrm{KWh}$ (mid-1990's) to mere $\$ 0.20 / K W h$ in 2008 . Market penetration of the PV industry increases steadily (under $1 G W$ in the US to $6 \mathrm{GW}$ by the year 2015). It is expected that the average KWh will be $\sim 10$ cents by or before 2015, with a steady GW plant installation. High efficiency solar cells $(\sim 50 \%)$ and parallel optical system advancement (total system at $30 \%)$, will lead to a very strong PV industry, for the benefit of all.

Current modeling of the top structure has indicated top efficiency values in excess of $21 \%$ (power out vs. power in) while for the bottom cell preliminary calculations indicate collection efficiency in excess of $25 \%$. The bottom cell is a GaAs-superlattice-Ge structure, where quantum size effects occur. Photo-excited carriers in the middle region are electrons trapped in quantum wells (thin germanium layers sandwiched by gallium arsenide layers). Thin Ge layers $(20 \mathrm{~nm})$ are tuned at $1 \mathrm{eV}$. They act as quantum traps and confine electrons in a discrete set of energy levels (one or two at the most). From these traps photo-electrons escape to the conduction band (minus the lost ones). Some advantages of our design over other high-efficiency full-spectrum solar cells are: (a) No excess tunnel junctions are needed to connect the cells (b) The superlattice region includes germanium layers tuned to absorb photons near $1 \mathrm{eV}$ (or more, depending on the quantum well thickness) (c) High mobility of carriers in both cells (top, bottom); the latter is a direct advantage over existing III-N-V high efficiency competing (nitrogen based) solar cell structures (d) Perfect lattice matching among the layers (e) Parallel carrier transport via (i) tunneling (ii) hopping and (iii) thermionic carrier escape. In the case at hand, tunneling is not a part of the action; instead thermionic emission currents are of importance. Maximum efficiency over $40 \%$ is expected via the synergistic action of the two cells.

\section{References}

[1] SM Sze, High-Speed Semiconductor Devices, John Wiley and Sons, 1990

[2] M Yamaguchi, Solar Energy Materials \& Solar Cells 90 (2006) 3068-3077

[3] M Yamaguchi et al, Solar Energy 82, 173 (2008)

[4] R. King, et al, 20th European PVSEC, 2005

[5] K Nishioka, Solar Energy Materials \& Solar Cells 90 (2006) 1308-1321, RR King, Nature Photonics, May 2008 
[6] Es Yang, Microelectronic Devices, McGraw-Hill, 1988

[7] JF Geisz, S Kurz, MW Wanlass, JS Ward, A Duda, DJ Friedman, JM Olson, WE McMahon, TE Moriarty, and JT Kiehl, Applied Phys. Letters 91, 023502 (2007)

[8] T Kirchartz, Uwe Rau, et al, Appl. Phys Lett. 92, 123502 (2008)

[9] T Mei, Journal of Appl Phys 102, 053708 (2007)

[10] AC Varonides and RA Spalletta, Physica Stat. Sol. 5, No. 2441 (2008)

[11] GFX Strobl at al, Proc. 7th European Space Power Conference, 9-13 May, Italy, 2005

[12] H. L. Cotal, D. R. Lillington, J. H. Ermer, R. R. King, S.R. Kurtz, D. J. Friedman, J. M. Olson, et al, 28th IEEE PVSC, 2000, p. 955

[13] T Kieliba, S Riepe, W Warta, Journal of Appl. Phys. 100, 093708 (2006)

[14] JF Geisz and DJ Friedman, Semicond. Sci. Technol. 17, 789 (2002)

[15] W Hant, IEEE Trans Electron Devices, VOL ED-26, NO 10, 1573 (1979)

[16] AC Varonides, Physics E 14, 142 (2002)

[17] E Istrate, EH Sargent, Rev Mod Phys, 78, 455 (2006)

[18] H E Runda et al, Nanoscale Res Lett (2006) 1:99

[19] W Li, BE Kardynal, et al, Appl. Phys Lett 93, 153503 (2008)

[20] AC Varonides, Thin Solid Films, Vol. 511-512, July 2006, pp 89-92

[21] BL Stein and ET Yu, Appl. Phys. Lett. 70 (25), 23 June, 1997

[22] AC Varonides, RA Spalletta, WA Berger, WREC-X and Exhibition, 19-25 July 2008, Glasgow, Scotland, UK.

[22] R. King, Multijunction Cells, Industry Perspective, Technology Focus, Nature photonics | VOL 2 | MAY 2008 | www.nature.com/naturephotonics

[23] T Kirchartz, BE Pieters, K Taretto, U Rau, Journal of Appl. Physics 104, 094513 (2008)

[24] AC Varonides and RA Spalletta, Thin Solid Films 516, 6729-6733 (2008)

[25] K Jandieri, S D Baranovskii, W Stolz, F Gebhard, W Guter,

[33] M Hermle and A W Bett, J. Phys. D: Appl. Phys. 42 (2009) 155101

[34] R Jones, CPV Summit, Spain, 2009 


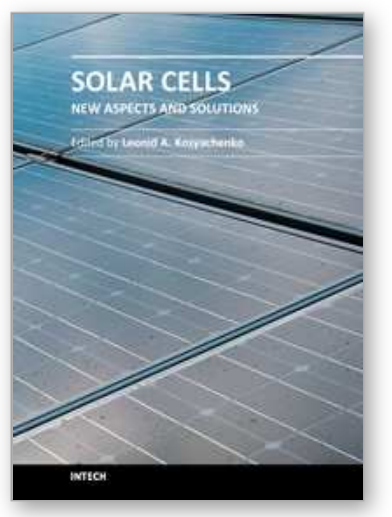

\author{
Solar Cells - New Aspects and Solutions \\ Edited by Prof. Leonid A. Kosyachenko
}

ISBN 978-953-307-761-1

Hard cover, 512 pages

Publisher InTech

Published online 02, November, 2011

Published in print edition November, 2011

The fourth book of the four-volume edition of 'Solar cells' consists chapters that are general in nature and not related specifically to the so-called photovoltaic generations, novel scientific ideas and technical solutions, which has not properly approved. General issues of the efficiency of solar cell and through hydrogen production in photoelectrochemical solar cell are discussed. Considerable attention is paid to the quantum-size effects in solar cells both in general and on specific examples of super-lattices, quantum dots, etc. New materials, such as cuprous oxide as an active material for solar cells, AlSb for use as an absorber layer in $p$-i-n junction solar cells, InGaAsN as a promising material for multi-junction tandem solar cells, InP in solar cells with MIS structures are discussed. Several chapters are devoted to the analysis of both status and perspective of organic photovoltaics such as polymer/fullerene solar cells, poly( $p$-phenylene-vinylene) derivatives, photovoltaic textiles, photovoltaic fibers, etc.

\title{
How to reference
}

In order to correctly reference this scholarly work, feel free to copy and paste the following:

AC Varonides (2011). High Efficiency Solar Cells via Tuned Superlattice Structures: Beyond 42.2\%, Solar Cells - New Aspects and Solutions, Prof. Leonid A. Kosyachenko (Ed.), ISBN: 978-953-307-761-1, InTech, Available from: http://www.intechopen.com/books/solar-cells-new-aspects-and-solutions/high-efficiency-solar-cells-viatuned-superlattice-structures-beyond-42-2-

\section{INTECH}

open science | open minds

\section{InTech Europe}

University Campus STeP Ri

Slavka Krautzeka 83/A

51000 Rijeka, Croatia

Phone: +385 (51) 770447

Fax: +385 (51) 686166

www.intechopen.com

\section{InTech China}

Unit 405, Office Block, Hotel Equatorial Shanghai

No.65, Yan An Road (West), Shanghai, 200040, China

中国上海市延安西路65号上海国际贵都大饭店办公楼 405 单元

Phone: +86-21-62489820

Fax: $+86-21-62489821$ 
(C) 2011 The Author(s). Licensee IntechOpen. This is an open access article distributed under the terms of the Creative Commons Attribution 3.0 License, which permits unrestricted use, distribution, and reproduction in any medium, provided the original work is properly cited. 